\title{
Tangence
}

\section{L'obsession de connaître (Beaulieu face à Melville)}

\section{Jean-François Chassay}

Numéro 41, octobre 1993

Interdiscurtivité dans l’œuvre de Victor-Lévy Beaulieu

URI : https://id.erudit.org/iderudit/025778ar

DOI : https://doi.org/10.7202/025778ar

Aller au sommaire du numéro

Éditeur(s)

Tangence

ISSN

0226-9554 (imprimé)

1710-0305 (numérique)

Découvrir la revue

Citer cet article

Chassay, J.-F. (1993). L'obsession de connaître (Beaulieu face à Melville).

Tangence, (41), 69-85. https://doi.org/10.7202/025778ar d'utilisation que vous pouvez consulter en ligne.

https://apropos.erudit.org/fr/usagers/politique-dutilisation/ 


\title{
L'obsession de connaître (Beaulieu face à Melville)
}

\author{
Jean-François Chassay *
}

Chaque vie est une encyclopédie, une bibliothèque, un inventaire d'objets, un échantillonnage de styles, où tout peut se mêler et se réorganiser de toutes les manières possibles.

Italo Calvino

Il y a certaines entreprises pour lesquelles un désordre soigneux est la vraie méthode.

Herman Melville

Ah Moby Dick! Ah maudit Bic!

Georges Perec

Dans Le poids du jour de Ringuet, publié en 1949, le principal protagoniste du roman, Michel Garneau, jeune provincial débarqué à Montréal, se lance en affaires à la suite de révélations sur sa naissance qui le décident à changer radicalement de vie. Comme plusieurs de ses semblables, il deviendra une véritable caricature du businessman américain. Le ridicule de la situation échappe pourtant constamment à ces hommes puisque, comme l'écrit le narrateur, "n'ayant jamais lu Babbit, ils ne se sentaient ni gênés, ni ridicules" 1 .

On ne peut pas dire qu'à cette époque les références explicites à la littérature américaine abondent dans le corpus québécois. Ce rappel du célèbre roman de Sinclair Lewis apparaît comme l'exception qui confirme la règle. Depuis, et surtout au cours des dernières années, elles se sont par contre multipliées: qu'on songe, pour ne prendre que quelques exemples, à Dashiell

* Jean-François Chassay est professeur à l'Université du Québec à Montréal.

1 Ringuet, Le poids du jour, Montréal, Variétés, 1949, p. 171. En ce qui concerne l'importance des États-Unis dans ce roman et l'idéologie qui s'y manifeste, on pourra lire mon texte intitulé "L'autre ville américaine. La présence américaine dans le roman montréalais (1945-1970)", Montréal imaginaire, Montréal, Fides, 1992, p. 279-322. 
Hammett dans Copies conformes de Monique LaRue, à Hemingway chez Jacques Poulin ou à Melville (une fois de plus) dans la trilogie des Mongols de Jean Basile. Cependant, chez aucun d'entre eux on ne retrouve l'obsession, avouée et affichée, que cherche à expliquer et à justifier l'écrivain-narrateur Abel Beauchemin dans ce Monsieur Melville dont le titre résonne déjà comme un hommage.

Auteur de La vraie saga des Beaucbemin, Abel affirme son rôle métalittéraire en "informant" sans cesse son œuvre des grands textes dont la lecture le touche et oriente son écriture: outre Moby Dick, Ulysse, Au-dessous du volcan, Don Quichotte sont notamment évoqués à plusieurs reprises. Mais dans l'ouvrage consacré à Melville, il y a davantage en jeu: l'auteur de Bartleby ne se contente pas (si on peut s'exprimer ainsi...) d'être un texte, il accède au rang de personnage, jouant un rôle central dans le développement diégétique. En ce sens Monsieur Melville, publié en 1978, s'inscrit de plain-pied dans une tradition relativement récente aux États-Unis, dont on voit se multiplier les exemples surtout depuis le début des années soixante-dix. Elle consiste chez les romanciers à utiliser des personnages publics - écrivains, philosophes, politiciens, artistes - dans leurs fictions, les situant dans un contexte souvent complètement étranger à celui dans lequel ils vécurent, ou interprétant de manière totalement subjective les événements auxquels ces individus ont participé 2. On ne doit pas s'étonner que les frontières apparaissent de plus

2 Parmi d'autres, notons Tatlin! (1974) et Da Vinci's Bicycle (1979) de Guy Davenport, The Public Burning (1977) de Robert Coover, Einstein's Dream (1991) d'Alan Lightman, Libra (1988) de Don Delillo, The World as I Found It (1987) de Bruce Duffy, The Adventures of Mao on the Long March (1971) de Frederic Tutten, Oxberding Tale (1982) de Charles Johnson. Il faut souligner aussi le cas très intéressant, mentionné par Marc Chénetier, de Genoa (1965) de Paul Metcalf qui est... l'arrière-petitfils d'Herman Melville. "Il porte [ce dernier] "comme un singe sur son dos", à la manière dont le "géant du passé" pesait sur les épaules de Hawthorne. Son Genoa est le prodigieux montage qui vise à l'en débarrasser." (Voir Marc Chénetier, Au-delà du soupçon. La nouvelle fiction américaine de 1960 à nos jours, Paris, Seuil, 1988, en particulier les pages 279-280). Beaulieu ne connaît pas (ou ne connaissait pas, à l'époque de la rédaction de Monsieur Melville) ce livre sans doute, puisqu'il n'en fait aucune mention. On se souviendra également que Saul Bellow apparaît comme personnage romanesque dans Volkswagen Blues de Jacques Poulin, mais il s'agit dans ce cas d'un rôle tout à fait secondaire dans l'économie du roman. 
en plus poreuses entre fiction et réalité dans une société où les communications (et à travers elles la publicité, les discours médiatiques) ont depuis fort longtemps des assises solides. Dans cet univers où la fiction repose de plus en plus sur l'image ${ }^{3}$, l'utilisation de personnages "réels" (souvent médiatiques d'ailleurs) dans le roman correspond dans bien des cas à ce que Régine Robin nomme le "postmodernisme $d u$ trouble".

Dans la littérature et dans l'art, dans la vie sociale, cette attitude ne vise ni à subvertir, ni à se résigner mais à contourner, à mettre à distance, à parodier, à déplacer, à décentrer de façon à susciter une inquiétante étrangeté, une attitude critique, de façon à faire des identités troublées, hybrides qui sont les nôtres aujourd'hui, une valeur positive, créatrice, et non inhibitrice. ${ }^{4}$

La complexité du livre de Victor-Lévy Beaulieu provient du fait que cette "lecture-fiction" n'est ni à proprement parler un roman, ni un essai. Fausse biographie sans être pour autant une biographie fictive (contrairement par exemple au Monde tel que je l'ai trouvé de Bruce Duffy, consacré à Wittgenstein), son "inquiétante étrangeté "tient à son indétermination générique aussi bien qu'à son cadre spatio-temporel. Il s'agit d'une quête de la connaissance où l'Histoire (américaine aussi bien que québécoise, les deux univers culturels apparaissant intimement liés à certains points de vue dans l'esprit du narrateur) joue un rôle extrêmement fécond.

Il va de soi que l'influence de Melville chez Victor-Lévy Beaulieu ne se limite pas à ce livre et l'importance de la culture américaine se manifeste dans sa prose, de manière implicite ou explicite, aussi bien dans Les voyageries que dans le reste de l'œuvre 5 . Dans Monsieur Melville par exemple, on apprend qu'Una, nom du personnage éponyme du roman suivant, était également celui

3 Le syntagme "Clinton for President" est devenu une fiction vraisemblable le jour où le futur résident de la Maison blanche s'est mis à jouer du saxophone devant les caméras d'une émission de télé. Nul doute que ceci a joué un rôle plus important pour les électeurs que sa vie matrimoniale, ses idées politiques ou son intérêt pour Cent ans de solitude, son livre préféré.

4 Régine Robin, "Postmodernisme, multiculturalisme et political correctness", Tangence, $\mathrm{n}^{\circ} 39$, mars 1993, p. 11 ( La fiction postmoderne").

5 Voir notamment l'article de Jonathan Weiss, "Victor-Lévy Beaulieu: écrivain américain ", Études françaises, vol. XTX, n 1 , printemps 1983, p. 4158 ("VLB"). 
72

d'un des enfants de Hawthorne ${ }^{6}$. Le réseau interdiscursif étatsunien s'étend ainsi des premiers livres jusqu'aux derniers. L'ampleur de Monsieur Melville cependant, son originalité et son importance stratégique dans l'univers beaulieusien méritent qu'on lui accorde une attention particulière.

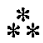

Étouffé peu à peu par l'influence de l'auteur de Moby Dick, Abel Beauchemin, en quelques pages qui sont parmi les plus belles publiées au Québec, convoque ses personnages et les congédie, le temps de se consacrer à Melville et de lui faire face: "À force de vivre avec l'autre, dans le monde des mots de l'autre, on en oublie sa puissance, on la fait devenir quotidienne, on la désamorce en quelque sorte, on y tue toute la beauté qu'on y avait vue et de laquelle on se nourrissait" (I, 11).

La métaphore de la nourriture et l'insistance sur l'existence de l'autre pour son propre développement, dans ce bref passage situé à la toute première page de Monsieur Melville, appellent déjà la notion d' "anthropophagie littéraire" dont se sert Jean Morency dans l'article qu'il publie dans ce numéro. Mais elles convoquent aussi l'idée du parasite, intéressante par sa polysémie, et dont a beaucoup traité Michel Serres ${ }^{7}$. William Paulson résume très bien la pensée du philosophe français:

The word parasite in French. denotes not only the organism that lives in or off another, not only the sponger who is always inviting himself to dinner, but also the static or noise that interrupts and muddles electronic communications. The word thus brings together biology, the anthropology of human relations, and communications theory, and this is no mere accident of language but a conceptual invariant: the parasite always interrupts, be it the circulation of nutritive elements, the service of food, or the transmission of signals. All three kinds of parasites have a parallel relation to order and disorder: they place themselves in relation to order that they have not pro-

6 Monsieur Melville, Montréal, VLB éditeur, 1978, vol. 2, p. 211. Les références seront dorénavant indiquées dans le texte entre parenthèses (le volume précédant la page).

7 Michel Serres, Le parasite, Paris, Grasset, 1980. 
duced, and their presence brings disorder to the systems in which they appear. ${ }^{8}$

Abel Beauchemin se perçoit lui-même comme un parasite, sentant le besoin de se regénérer auprès d'un organisme hôte pendant une phase de son cycle vital. Les voyageries, pour continuer à se développer et pouvoir se rendre à terme, exigent la présence de Melville. Beauchemin souffre de cette nécessité, se percevant comme un usurpateur. Pourtant, au delà de la dimension psychologique par laquelle il situe toujours son travail à l'aune de celui de Melville, le livre n'a de sens que dans la mesure où son ouvre vient sans cesse court-circuiter celle de l'écrivain américain; elle essaime comme autant de bruits qui viennent interrompre le cours régulier de la chronologie et de sa linéarité, plaçant sans cesse le lecteur en position "d'attente de l'imprévu "?. Le déroulement événementiel se voit sans cesse paralysé par les propos du narrateur qui déplace le centre d'intérêt.

En réalité, Beaulieu inverse le propos biographique traditionnel. En principe, on écrit une biographie dans le but de rendre compte d'une "vie bien remplie" et le texte vise à montrer à la fois le déroulement de celle-ci et l'intérêt pour les générations futures de celui dont on dessine le portrait. Mais l'existence de Melville, arrimée à celle de Beauchemin, se voit continuellement relancée par la vie et l'œuvre de ce dernier, comme si elle ne suffisait pas à combler les désirs d'écriture du narrateur. D'ailleurs, dans une formule toute flaubertienne - Flaubert est d'ailleurs une autre des figures qui dominent ce livre et les comparaisons ne manquent pas entre les deux écrivains, aussi bien en ce qui concerne leur vie que leur production -, Beauchemin affirme: "Mais je suis bien obligé d'avouer que ce projet ne repose sur rien, que sur ma vie que j'aimerais faire éclater pour ainsi dire définitivement" (I,22). Voilà Melville, à qui ce livre doit être consacré, bizarrement renvoyé dans les limbes.

L'intérêt du projet beaulieusien tient pour une bonne part à ce paradoxe, et l'épigraphe qui ouvre le deuxième volume - "Il

8 William Paulson, The Noise of Culture, New York, Cornell University Press, 1988, p. 37.

9 Pour reprendre une formule d'Umberto Eco: "Dans la réception d'un message structuré de façon ouverte, l'attente implique moins une prévision de l'attendu qu'une attente de l'imprévu. " (L'œuore ouverte, Paris, Seuil, coll. "Points", 1979, p. 105). 
y a certaines entreprises pour lesquelles un désordre soigneux est la vraie méthode" - indique bien que le sens n'est jamais acquis, qu'il doit surgir de ces télescopages, de ces incertitudes face au foisonnement de l'œuvre, de cette permanente entropie qui permet au narrateur d'écrire qu'il se voit comme le "maître de l'énergie" (I, 132), véritable démon de Maxwell, seul capable d'équilibrer et d'ordonner les signes épars de ce texte en devenir. Toutefois, cela ne signifie pas pour autant que les choses soient plus simples pour lui et les fils de la narration viendront souvent près de se rompre.

Une fois le dernier mot écrit, le roman donnerait l'impression de se tenir dans tous ses morceaux, on y devinerait une intention précise, une chronologie et un temps particuliers, comme si le livre ne pouvait pas être différent de ce que je l'avais fait devenir. (I, 13)

Illusion naïve évidemment, puisque "la vérité ne peut être qu'absolument autre: j'avais choisi cela mais tout autre chose aurait pu survenir" (I, 13). C'est bien d'abord cette tentative de mise en forme d'une matière sans cesse aléatoire, reprise et remise en question, que propose Monsieur Melville et à laquelle s'acharne, parfois désespérément, le narrateur.

Essai sur Melville, le livre de Victor-Lévy Beaulieu s'ouvre d'emblée sur la fiction ${ }^{10}$, tout en proposant une réflexion sur le vrai et le vraisemblable: "Bien sûr, tout livre qu'on écrit sur quelqu'un d'autre que soi est un prétexte. Mais soi-même, on est aussi un prétexte. De quoi rendre compte alors? De quel lieu qui en soi est le vrai?" (I, 19) Quel ancrage en effet donner à la (sa) réalité? Quel poids peut-on accorder au mot "réel "?

La pertinence de cette question ne fait pas de doute dans l'ouvrage, dans la mesure où le lecteur se voit sans cesse balloté

10 On notera par ailleurs que Moby Dick, livre autour duquel et à partir duquel s'écrit Monsieur Melville - les sous-titres des trois volumes en font foi -, bien qu'authentique roman, ne manque pas d'ambiguités sur le plan générique. Non seulement de nombreux passages sont consacrés à l'histoire des baleines, aux mythologies les entourant et au travail des baleiniers, donnant parfois l'impression au lecteur d'être plongé dans un essai, mais de plus on retrouve parfois des notes en bas de page, sans aucune marque d'ironie (contrairement à ce qu'on peut lire dans L'biver de force par exemple). Cette présence surprenante provoque un effet antiromanesque qui vient accentuer l'impression ressentie, à partir du moment où le Pequod prend la mer, que nous sommes parfois au cour d'un essai. 
entre "la fiction et ses doubles", pour reprendre une formule de Lucien Dällenbach ${ }^{11}$. En effet, soumis à diverses manifestations de mise en abyme, Monsieur Melville provoque l'obsolescence de l'idée même de réalité. La fiction renvoie à elle-même et se mord la queue.

Plusieurs éléments d'ordre biographique rapprochent le personnage de l'écrivain Abel Beauchemin de Victor-Lévy Beaulieu lui-même, au point de pousser, au fil des ans, de nombreux critiques à voir dans le premier un double du second, sinon à les confondre carrément. Cette oscillation entre réel et fiction est fortement complexifiée dans Monsieur Melville.

Abel Beauchemin, personnage fictif, bat le rappel de ses personnages (nécessairement fictifs) pour leur signifier leur congé, le temps de rédiger un ouvrage sur un auteur (de fiction) dont l'existence au $\mathrm{XIX}^{\mathrm{e}}$ siècle ne peut être remise en question. Et pourtant, selon le narrateur, ce livre en cours ne peut s'écrire comme une biographie, c'est-à-dire à partir de faits (supposément) objectifs: "C'est pourquoi Melville, tout comme moi, ne peut que basculer du côté de la fiction" (I, 23). Le temps de l'écriture, l'écrivain "réel" et l'écrivain fictif vont devenir les personnages d'une fiction, celle que le narrateur est en train d'écrire (à partir d'éléments qu'il peut colliger, dans des textes, concernant l'existence de Melville). Mais si l'écrivain américain devient un personnage du livre en cours de Beauchemin, il plonge également ce dernier dans sa propre fiction. Le baptisant du nom de Hawthorne (grand ami de Melville lui-même comme on le sait), il le projette dans l'univers de Moby Dick. Les chapitres trois et quatre de Moby Dick sont repris partiellement, mais avec de sérieuses modifications. Voilà que Queequeg ne dort plus avec Ishmaël mais bien avec Melville lui-même, en plus du narrateur du texte qu'on a sous les yeux (disons, pour simplifier: Victor-Lévy Beaulieu, alias Abel Beauchemin, alias Nathaniel Hawthorne, alias un nouveau personnage de Moby Dick), lui-même emporté par la fiction de celui dont il veut écrire la biographie, modifiant certains éléments de Moby Dick de l'intérieur. À cela, il convient d'ajouter que les personnages des romans de Beauchemin font régulièrement leur apparition dans Monsieur Melville. On comprendra après cela que le narrateur puisse écrire une phrase aussi

11 Voir Lucien Dällenbach, Le récit spéculaire, Paris, Seuil, 1977, p. 76. 
étonnante que «Dans ma fiction, je ne retrouve aucun fait qui confirmerait le propos de Giono. (III, 15), ce dernier ayant luimême émis des hypothèses audacieuses concernant certaines motivations de Melville qui n'ont pu être confirmées ${ }^{12}$. C'est comme si, à travers cette véritable pulvérisation de la réalité qu'est Monsieur Melville, seule la fiction, dans sa quête aléatoire d'un univers qui ne se justifie jamais, pouvait donner sens et vraisemblance à une réalité s'affichant comme véridique ${ }^{13}$.

Assistant à la production d'un texte qui réfléchit sur lui-même et se réfléchit, le lecteur suit un mouvement oscillatoire qui rend difficile la distinction entre récit enchâssé et récit enchâssant, l'œuvre de Beauchemin et celle de Melville se répondant comme des vases communicants. Or, comme l'écrit Dallenbäch,

[...] toute "histoire dans l'histoire", en tant que réflexive, est nécessairement conduite à contester le déroulement chronologique qu'elle respecte en tant que segment narratif. [...] incapable de dire la même chose en même temps qu'elle, l'analogon de la fiction, en le disant ailleurs, le dit à contretemps et sabote par là même l'avancée successive du récit ${ }^{14}$.

C'est à cela que nous convie ici l'auteur, mais dans un livre, qui plus est, qu'on ne peut appeler au sens strict une fiction et où se multiplient les dérapages. Le narrateur écrit par exemple, insistant sur l'importance du regard qui permet à la fiction d'advenir :

C'est grâce à mes yeux que je vais remonter à la surface de Moby Dick, tirant par eux la moëlle de toutes ses créatures que j'ai inventées pour ne pas être seul - sans doute comme j'ai inventé Melville et fait apparaître Moby Dick, parce que je suis odieux et désespéré par ma vie. (II, 274)

Il s'agit d'une phrase symptomatique en ce qu'elle provoque une série de télescopages visant à abolir la linéarité du récit:

12 "Sur le plan de la représentation, Giono fait de Melville, en dessinant son portrait, rien de moins qu'un autre Giono. (III, 13). Beauchemin ne fait pas autre chose, comme il l'affirme lui-même. L'auteur du Grand troupeau a opéré de la même manière que le futur auteur de La grande tribu.

13 Beauchemin le dira autrement en poussant au bout de sa logique certaines idées de Hawthorne: "Rencontre du réel et de l'imaginaire, dit Hawthorne qui n'ose pourtant pas aller jusqu'au bout de sa pensée: une fois débarrassé de ses stéréotypes, le réel n'est que de l'imaginaire" (II, 196).

14 Lucien Dallenbäch, Le récit spéculaire, op. cit., p. 82. 
Melville et son œuvre renvoient aux personnages créés par Beauchemin dans ses livres, ce dernier les justifiant par ses problèmes existentiels. Ce genre de dérapage s'affirme comme une des constantes de l'ouvrage. Les commentaires du narrateur louvoient ainsi entre connaissance et reconnaissance, frustration et jubilation, cherchant dans les méandres de l'œuvre melvillienne un sens qui ne peut se trouver dans une simpliste progression linéaire. À maintes reprises, Beauchemin lui-même rend compte de ces dérapages comme s'il ne pouvait les contrôler: "Je dois paraître m'égarer" (I, 74); "Je crains fort de m'être égaré quelque part le long du chemin" (I, 94-95); "je ne sais pas pourquoi j'écris déjà cela" (I, 140); etc. Ou encore ceci, passage sur lequel il faudra revenir et qui justifierait, selon le narrateur, son manque de contrôle: "Cette lecture de Melville m'a bouleversé et j'en ai perdu les pédales. Je ne saurai jamais être chronologique. Bien trop québécois, c'est-à-dire privé de temps" (III, 64).

Faisant fi de la chronologie, Beauchemin revient sans cesse à Moby Dick ("une grande fièvre m'habite et me pousse à laisser tomber toutes les balises pour ne parler que de Moby Dick" (I, 103)), anticipant l'écriture de ce livre et se servant de celui-ci pour expliquer ce qui va suivre, provoquant ainsi des effets de feedback ${ }^{15}$ constants: tournoyant autour de ce roman central, le livre n'y échappera qu'au cours du dernier volume, lorsque la fin de la rédaction de Pierre permet de se soustraire à l'emprise de la baleine blanche. "Maintenant, cela va aller très vite, un certain Melville vient de disparaître à jamais" (III, 39). Reste que même après cela, l'emballement provoqué par la structure chaotique de l'ouvrage ne s'imterrompt pas pour autant.

Tout au long de la narration, Beauchemin s'extrait de son travail sur Melville pour revenir à des préoccupations personnelles, s'intéresser à ce que disent ou pensent ses personnages. Les chapitres s'ouvrent régulièrement sur des pauses qui sont plus souvent qu'autrement des propos réitérés sur l'écriture, qui en relancent le mouvement, comme si nous étions dans un éternel recommencement: "Ainsi donc vais-je dans mon livre sur Melville,

15 Rappelons que le feedback en cybernétique signifie "l'autorégulation qui règle le jeu des écarts rétroactivement par rapport au circuit: on peut par extension désigner ainsi l'intégration quasi automatique d'une information présente dans un système de sens préalable en voie de constitution" (Michel Picard, Lire le temps, Paris, Minuit, 1989, p. 27). 
plaçant une à une les pièces que je choisis, peu pressé d'en finir, comme si je n'osais pas vraiment aller au cœur du problème" (I, 103); "Je reste obstinément devant la table de pommier, les yeux fermés, songeant à tout ce que j'ai écrit, n'essayant pas toutefois d'ameuter la suite " (I, 159); "On ne s'imagine pas comme c'est long écrire: toutes ces heures qu'on passe assis devant la table de pommier, à aligner toutes sortes de mots et tant de ratures parce qu'on se sent extrêmement maladroit, impuissant à dire le meilleur de Melville [...]" (II, 55).

Cette impression de reprises et de recommencements est accentuée par une série de redondances qui viennent atténuer l'entropie du discours, comme cette obsédante première image, "l'image la plus tenace que j'ai de Melville" (I, 25) écrit le narrateur, celle d'un homme âgé, au seuil de la mort. C'est sur cette image de la fin que se développent les premières réflexions sur l'écrivain, et le livre ne peut qu'y reconduire à mesure qu'il s'approche de son achèvement. D'entrée de jeu, Beauchemin indique que cette structure prend sa source dans l'acte même d'écrire: "Écrire ne constitue pas une orientation parce que cela ne commence ni ne s'achève, parce que cela ne fait que se recommencer pour occuper tout le champ de ses fissures et, par cela même, en produire de nouvelles, et d'autres encore, jusqu'à l'extinction de soi" (I, 14).

Tout ceci s'ajoutant à l'identification, même physique, des personnages entre eux - Beauchemin métamorphosé en Melville, celui-ci devenant Ishmaël, Samm prenant soudainement la place d'Augusta - ou encore des espaces - Arrowhead devenant la Mattavinie - conduit à un éclatement du cadre spatiotemporel et même, pourrait-on dire, à une abolition du temps. Il s'agit d'un aspect central du travail de Beaulieu.

Abel Beauchemin constate avec surprise que son père lui parle de Melville comme s'il s'agissait de l'un de ses amis, "comme si nous étions du même âge" (I, 94). Cette impression corrobore pourtant celle du lecteur devant ces pages où les vies s'emboîtent les unes dans les autres et où les personnages surgissent comme autant de palimpsestes qui permettent de réécrire et de réinterpréter les événements, au gré des points de vue, en faisant fi de la réalité du temps. Il n'y a pas de quoi s'en étonner: le narrateur affirme bel et bien "qu'il vient un temps où tout se prend comme dans un pain, curieuse force centrifuge qui fait qu'on est toujours dans le présent, virtuellement actuel, dans le cercle de soi-même, avec tout ce qui fut et continuc d'être, sauf 
que ça se produit désormais sans mémoire, dans la permanence de l'épiphanie où tout, en même temps, jaillit avec force et sans que ça puisse se retenir" ( $I, 38$ ) (l'ombre de Joyce, comme toujours chez Beaulieu, hante ses pages).

Tout au long du livre, comme dans la citation qui précède, le temps n'existe que dans l'instant, échappant à la flèche qui va du passé au futur ${ }^{16}$. C'est dans ce présent inaltérable, cette immobilité invariable, que la figure de Melville apparaît le plus clairement dans toute sa solitude (qui est aussi celle de Whitman et de Dickinson, à la même époque): "Ce n'est pas le personnage qu'il cherche mais lui-même. Ce n'est pas au mouvement qu'il aspire mais à l'immobilité " (I, 53); "À dire vrai, tout était immobile quand on savait déconstruire le mouvement apparent et aller audelà de ce qui paraissait l'agiter" (I, 82); "Celui qu'on a enfermé [parlant de Bartleby], c'est l'immobilité même" (III, 59). Beauchemin s'y retrouve, lui qui va jusqu'à souhaiter que le temps de l'écriture - et l'idée de durée, qui lui est consubstantielle s'abolisse: "Alors on atteindrait un autre état, enfin non linéaire, qui ferait éclater la phrase et l'ouvrirait comme un ventre femelle, de quoi féconder le livre ou ce que serait devenu le livre, c'est-àdire une prodigieuse énergie intemporelle» (I, 119).

Cette énergie première, fondamentale, irradiante, vient prendre le contrepied du temps linéaire et crée également des interférences dans le texte. Entre le temps qui s'écoule, lié à l'avancée de l'écriture, à la connaissance croissante de l'œuvre melvillienne, et les événements qui se produisent dans le livre,

16 La question du temps, étudiée d'un point de vue neurologique, pemet de rapprocher, une fois de plus, Melville et Flaubert. On sait que les deux hommes étaient atteints d'une "maladie nerveuse". La nature de celle-ci chez Flaubert a provoqué des interprétations nombreuses et variées (voir les "Notes et variantes" établies par Jean Bruneau, Gustave Flaubert, Correspondance I, Paris, Gallimard, "Bibliothèque de la Pléiade", 1973, p. 943-944). Paul Virilio s'intéresse quant à lui aux "picnoleptiques", dont font partie les épileptiques. Le mot cependant a un sens beaucoup plus large et désigne, comme dans le cas de personnes atteintes de maladie nerveuse mais sans que ce soit exclusif, ceux qui sont victimes "d'absences". Mais pour le picnoleptique, rien non plus ne s'est passé, le temps absent n'a pas existé; à chaque crise, sans qu'il s'en doute, un peu de sa durée lui a simplement échappé. (Paul Virilio, Estbétique de la disparition, Paris, Galilée, 1980, p. 14). De ce point de vue, Melville et Flaubert, à cause de leur condition physique, seraient des écrivains pour qui la notion de temps serait singulière. 
80

appartenant souvent à "un monde qui n'obéit pas au temps et à l'espace" (I, 127) tels qu'on peut les concevoir, il existe un hiatus. L'irréversibilité du temps associé au devenir biologique, résultante de la structure de l'esprit humain qui fonctionne selon une logique de cause à effet, ne correspond pas au "temps" de l'univers, où passé et futur ne veulent rien dire. "Une théorie psychologique et une théorie cosmologique du temps s'occultent réciproquement, dans la mesure même où elles s'impliquent l'une l'autre." 17 Depuis Parménide, ce problème philosophique a occupé les esprits. La conciliation impossible entre l'idée d'infini comme espace et temps absolus et notre connaissance empirique de l'espace et du temps constitue le motif de nombreux romans contemporains ${ }^{18}$. Que signifie-t-elle dans Monsieur Melville? On pourrait avancer qu'elle sert à interroger l'Histoire et ses savoirs ainsi que le rôle de celle-ci dans la fiction.

Retraçant l'adolescence de Melville, Beauchemin l'imagine en compagnie de son oncle Peter, ce dernier pestant devant les malheurs de la famille. Comparant la présidence d'Andrew Jackson à celle de John Quincey Adams, il affirme: "Si toute l'Amérique vivait comme a vécu Adams, il n'y aurait pas cette décadence qui risque de tuer définitivement l'esprit des Pères fondateurs" (I, 112).

Cette nostalgie caractéristique par laquelle le passé peut être figé, statufié et par le fait même apprécié parce que dégagé du bruit et de la fureur des événements, représente justement ce contre quoi s'acharne le texte littéraire. La nostalgie est la mort du passé, et par la même occasion la mort de l'Histoire. La littérature ne peut qu'être l'expression d'une Histoire en mouvement, tournoyant sur elle-même et embrassant dans sa trajectoire la réalité du lecteur. S'arrêtant sur l'œuvre de Kafka, Hannah Arendt s'interroge justement sur la temporalité, à partir de la fiction:

Dans les mots de Faulkner, "le passé n'est jamais mort, il n'est même pas passén. Ce passé, en outre, dont la portée s'étend jusqu'à l'origine, ne tire pas en arrière mais pousse en avant, et c'est contrairement à ce que l'on attendrait, le futur qui nous repousse dans le passé. Du point de vue de l'homme, qui vit

17 Paul Ricœur, La configuration dans le rëcit de fiction, Paris, Seuil, 1984, p. 22.

18 Dans la production récente, on peut penser à l'exemple du jeune bomme, remarquable roman de Botho Strauss dont on a très reu parlé au Québec (Paris, Gallimard, 1986). 
toujours dans l'intervalle entre le passé et le futur, le temps n'est pas un continuum, un flux ininterrompu; il est brisé au milieu, au point où "il" se tient; et "son" lieu n'est pas le présent tel que nous le comprenons habituellement mais plutôt une brèche dans le temps que "son" constant combat, "sa " résistance au passé et au futur fait exister. ${ }^{19}$

"L'immobilité" propre à Melville qui n'est peut-être que ce point où il se tient, Beauchemin la retrouve dans le Québec qu'il habite, lieu "privé de temps" (III, 64), "petit état équivoque qui ne peut trouver ses appuis dans l'histoire, sinon pour se percevoir comme colonisé, ce qui ramène tout de suite la notion d'absence et celle de l'immobilité" (I, 221). Face à une littérature nationale en train de se constituer, à l'époque de Melville, Hawthorne et Whitman, Beauchemin ne peut manquer d'évoquer des parallèles avec le Québec récent. Il se projette dans ce passé américain, y cherchant les traces de son environnement culturel, conscient que les coïncidences entre les deux univers ne peuvent masquer tout ce qui les sépare.

Certes, il y a là un discours qui relève du politique et du mythique face à une (future) grande littérature alors en émergence. Bien sûr, la comparaison ne peut être que cruelle:

Mais moi, je n'ai jamais commencé [...]. Je sombre et ce ne sera toujours que cela, une chute sans fin dans les eaux du non-être: il n’y a ni temps, ni espace québécois, que de la présence américaine [...]. Américain mais sans l'Amérique, consommateur mais sans capital, esclave de l'Empire et sans d'autres armes que ce pitoyable livre pour me continuer dans ma pâle énergie (III, 126).

Plus fondamentalement cependant, au delà de ce qui pourrait apparaître comme une longue lamentation, ses réseaux de coïncidences conduisent à interroger le savoir même du texte fictionnel à l'intérieur du grand texte historique.

Dans l'histoire, la littérature vient [...] sans cesse opposer son chaos et ses fluidités à tout ce qui ailleurs tente de prendre en prenant forme, et si l'histoire vue par les historiens se meut avec lenteur, la littérature qui l'accompagne évoque plutôt les ciels de cinéma vus en accéléré: ils nous révèlent derrière les plus paisibles nuages de silencieuses tempêtes. ${ }^{20}$

19 Hannah Arendt, La crise de la culture, Paris, Gallimard, coll. "Folio", 1989, p. 21-22.

20 Michel Pierssens, Savoirs à l'ouvre, Lille, Presses universitaires de Lille, 1990 , p. 13-14. 
82

Chaos et fluidité: voilà deux termes qui conviennent parfaitement à cette narration en spirale, tourbillonnante, dont la forme exprime d'abord un refus des certitudes trop facilement placardées.

Si Beauchemin prend plaisir à citer Melville critiquant Washington Irving, c'est parce que celui-ci ne fait qu'imiter le passé, un passé figé et qui n'apprend rien alors que Hawthorne, au contraire, plonge dans le passé pour le réinventer et en faire une authentique tragédie moderne. De même, "Poe et Whitman sont de véritables écrivains [parce que] leurs livres ajoutent quelque chose de fondamental à la culture déjà existante" (II, 101). Et qu'est-ce que cet ajout, sinon l'irruption au départ d'une question fondamentale: "que sait, que suggère cette œuvre qui n'aurait pas été compris ou entrevu avant elle?"21 Autrement dit, dans le flot des connaissances actuelles, comment la littérature, comme discours du savoir, peut-elle se distinguer?

Dès les premières pages du livre, Beauchemin situe au centre du travail de l'écriture la question de la connaissance: "je fouille partout et je n'arrive pas à faire une connaissance de mon savoir. C'est pourtant tout ce qui donne son sens à l'écriture" (I, 20). Toute la prose du livre est d'abord une quête de la connaissance, quête quasi alchimique - on connaît l'importance du sacré chez Beaulieu - non pas de la vérité mais d'une certaine cohérence qui passe à travers l'Amérique étatsunienne, focalisée sur la figure d'un homme, Herman Melville, qui naît et vit à un tournant de l'histoire du pays. Mais cette quête ne peut aboutir que s'il y a un espace qui permette de la penser. Or Beauchemin, dans son "petit pays équivoque" (III, 128), se voit comme "un moins que rien, une prose dont le lieu ne s'appartient pas" (III, 128), condamné à errer, à rejoindre ceux qui, comme Melville, ne trouvent pas leur place et restent, bon gré mal gré, "des desesperados de l'Amérique" (III, 64).

Comment donc atteindre à la connaissance à travers la fiction? Relevant des titres d'œuvres qui lui paraissent fondamentales, Beauchemin écrit qu'il s'agit de créations totalisantes "dont l'écriture pose la vraie question: le savoir peut-il se transformer en connaissance? Après les cuvres de James Joyce, d'Herman

21 William Paulson, "Épistémocritique et écologie culturelle", TLE, no 10 , automne 1992, p. 126 ("Épistémocritique et cognition"). 
Broch, de Gustave Flaubert et de William Gaddis, on peut sans doute prendre tout cela avec un grain de sel " (II, 120). Il faut au contraire s'arrêter sur ce "grain de sel ". La question de la connaissance dans la fiction se révèle aujourd'hui centrale et un livre comme Monsieur Melville apparait justement comme une tentative de réponse.

Curieusement, les analyses des textes melvilliens par Beauchemin représentent sans doute la partie la moins intéressante, la plus scolaire de l'ouvrage de Beaulieu, alors qu'on pouvait s'attendre au contraire à ce que les interprétations qu'il en donne permettent d'apporter une certaine connaissance au lecteur. Si elles ennuient plutôt, c'est parce qu'elles figent les textes, échappant au mouvement qui parcourt le livre, comme si, par respect excessif, il fallait éviter les bruits, les parasites, qui risqueraient d'indisposer le lecteur. Les lectures se limitent fréquemment à la paraphrase et font preuve bien souvent d'un académisme (la passion en plus néanmoins) qui déçoit.

Au contraire, l'image qui se fixe peu à peu de Melville (tout en restant un peu floue), la réflexion sur sa poétique que Beauchemin prend peu ou prou à son compte et le portrait qui se dessine de l'Amérique sont prodigieusement libres. Contrairement aux penseurs mous qui clament de manière névrotique la fin de l'Histoire, Beaulieu place celle-ci au centre de son propos, la prend à bras le corps, en en faisant le cœur d'un nouveau "grand récit" de l'Amérique. Monsieur Melville se propose alors comme "roman contemporain en tant qu'encyclopédie, en tant que moyen de connaissance, et surtout en tant que réseau reliant les faits, les personnes et les choses" 22 , procédant par tâtonnements et reprises, offrant l'image même d'une réflexion continue, se déplaçant sans cesse.

Ouverte, l'ouvre d'Abel Beauchemin ne peut imposer une clôture qui signifierait par la même occasion la fin de la lecture et la fin de l'Histoire. Elle relance l'une et l'autre en créant de nouveaux points de jonction. Melville est un point de référence qui permet de faire communiquer des éléments selon des modalités particulières au texte littéraire.

En un temps où d'autres media triomphent, dotés d'une vitesse très élevée et d'un rayon d'action très étendu, menaçant de

22 Italo Calvino, Leçons américaines, Paris, Seuil, 1989, p. 169-170. 
84

réduire toute communication à une croûte uniforme et homogène, la fonction de la littérature est de faire communiquer le divers avec le divers comme tel; sans émousser sa différence, mais en l'exaltant au contraire, selon la vocation du langage écrit.

Le siècle de la motorisation a imposé la vitesse comme valeur mesurable [...] mais la vitesse mentale ne peut être mesurée et ne permet ni comparaisons ni compétitions. ${ }^{23}$

C'est grâce à cette vitesse que le temps peut être aboli dans le texte, au détriment du réalisme (mais qu'importe). Ce qui compte avant tout, c'est la façon dont la pensée peut se projeter dans le temps à une époque, la nôtre, où "le développement des hautes vitesses techniques [pourrait aboutir] à la disparition de la conscience en tant que perception directe des phénomènes qui nous renseignent sur notre propre existence "24. Situé au Québec, ce point aveugle de l'Amérique, "cette quantité négligeable que mêtme l'énorme Pablo Neruda a confondu avec l'Amérique saxonne jetant ses bombes au napalm au-dessus du Vietnam" (III, 63), Abel Beauchemin projette sa conscience à travers l'espace et le temps pour exprimer toute la négativité liée à ce territoire qu'il habite et qui pourtant n'existe pas vraiment. Encore faut-il rendre compte de tout cela formellement. À travers Monsieur Melville, "c'est cette mobilité de la trajectoire synoptique qui, en modifiant le point de vue du sujet, va lui permettre la découverte de ce qui, d'une certaine manière, était à portée du regard 25 .

La lecture de l'œuvre melvilienne provoque l'interaction de deux discours, celui de l'écrivain américain et d'Abel Beauchemin qui se confondent peu à peu. Monsieur Melville démontre, davantage encore que les autres livres de Beaulieu, qu'un texte ne s'écrit jamais seul. Paradoxalement, l'identification de plus en plus forte d'Abel à son maître littéraire devient une dérive qui, par rétroaction, permet au narrateur de renouer les fils épars de sa propre ouvre. C'est ainsi que les "bruits" qui viennent brouiller la communication, deviennent, à un autre niveau, de l'information, puisqu'ils permettent peu à peu de définir une poétique qui prend forme sous les yeux du lecteur.

Quant à la cruelle comparaison entre les États-Unis et le Québec, entre la réalité de plus en plus forte du premier et

23 Ibid., p. 80-81.

24 Paul Virilio, Esthétique de la disparition, op. cit., p. 119.

25 Ibid., p. 123. 
l'inexistence maintes fois réitérée du second, on peut la relativiser grâce à Melville en relisant Moby Dick: "Queequeg was a native of Kokovoko, an island far away to the West and South. It is not down in any map; true places never are. " 26

26 Herman Melville, Moby Dick, New York, New American Library, "A Signet Classic", 1961, p. 70. 